\title{
2-NILPOTENT AND RIGID FINITE DIMENSIONAL ALGEBRAS
}

\section{CLAUDE CIBILS}

\section{Introduction}

Let $k$ be a field and let $\Lambda$ be a finite dimensional $k$-algebra. We suppose that $\Lambda$ is Morita reduced and that the endomorphism ring of each simple $\Lambda$-module is $k$. This is equivalent to $\Lambda / r=k \times \ldots \times k$, where $r$ is the Jacobson radical of $\Lambda$.

Let $Q$ be the Gabriel quiver of $\Lambda$ which is defined as follows: the set $Q_{0}$ of vertices is in one-to-one correspondence with the set of isomorphism classes of simple $\Lambda$-modules. If a simple $\Lambda$-module is denoted by $S$, we denote by $s$ the corresponding vertex. The number of arrows from $s$ to $t$ is the $k$-dimension of $\operatorname{Ext} \lambda(S, T)$. The set $Q_{1}$ of arrows of the quiver is the disjoint union of the set $B$ of loops and the set $A$ of oriented edges.

Let $k Q$ be the quiver algebra. By an observation of Gabriel $[4 ; 5,4.3]$ we have an algebra surjection $k Q \rightarrow \Lambda$ whose kernel $I$ is admissible, that is, one has $F^{n} \subset I \subset F^{2}$, where $F$ is the two sided ideal of $k Q$ generated by the arrows, and $n$ is some positive integer.

In this way each finite dimensional $k$-algebra $\Lambda$ such that $\Lambda / r=k \times \ldots \times k$ is isomorphic to $k Q / I$ with $I$ admissible. The radical $r$ of $k Q / I$ is $F / I$.

By definition the algebra $\Lambda$ is 2-nilpotent if $r^{2}=0$. In the previous description this is equivalent to $I=F^{2}$.

Let $H^{*}(\Lambda, X)$ denote the Hochschild cohomology groups of $\Lambda$ with coefficients in the $\Lambda$-bimodule $X$ (see [2] for a definition).

In this note we give a description of the algebras $\Lambda=k Q / F^{2}$ such that $H^{2}(\Lambda, \Lambda)=0$. Supposing $Q$ is connected, the precise conditions are that $Q$ does not contain loops nor unoriented triangles

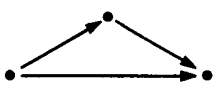

and $Q$ is not the quiver $\rightleftarrows \bullet$.

Let us recall that for hereditary algebras, $H^{2}(k Q, X)=0$ for all quivers $Q$ without oriented cycles and all $k Q$-bimodules $X$, see $[6,3]$.

If $k$ is algebraically closed, the orbit of a $k$-algebra $\Lambda$ of dimension $n$ under the natural action of $\mathrm{GL}_{n}$ is open (in the scheme sense) if and only if $H^{2}(\Lambda, \Lambda)=0$ and the algebraic group Aut $\Lambda$ of automorphisms of $\Lambda$ is smooth. When the characteristic is zero, the orbit of $\Lambda$ is open if and only if $H^{2}(\Lambda, \Lambda)=0$, because then the algebraic groups are smooth (see [6]).

In [7] Gerstenhaber shows that if $H^{2}(\Lambda, \Lambda)=0$, then $\Lambda$ is rigid, that is, each

Received 19 May 1986; revised 30 September 1986.

1980 Mathematics Subject Classification 16A46 and 16A61.

Supported by a FNSRS (Swiss National Science Foundation) grant.

J. London Math. Soc. (2) 36 (1987) 211-218 
one-parameter family of deformations of $\Lambda$ is trivial. We shall prove directly that the given conditions on $Q$ imply that $\Lambda=k Q / F^{2}$ is rigid. I thank the referee for suggesting this proof, which is shorter than the original one.

The converse of the result is proved using the fact that a connecting homomorphism in a long exact sequence of cohomology groups is a cup product with a fixed element.

In [3] we gave sufficient conditions on the quiver $Q$ and the ideal $I$ of a finite dimensional algebra $\Lambda$ for $H^{2}(\Lambda, \Lambda)$ to be zero. However these conditions are not necessary as this note shows; see the remark at the end.

Finally we remark that it is not hard to show that

$$
\operatorname{dim}_{k} H^{\mathrm{I}}(\Lambda, \Lambda)= \begin{cases}\sum_{s, t \in Q_{0}}\left|t Q_{1} s\right|^{2}-\left|Q_{0}\right|+1 & \text { if char } \mathrm{k} \neq 2, \\ \sum_{s, t \in Q_{0}}\left|t Q_{1} s\right|^{2}-\left|Q_{0}\right|+1+|B| & \text { if char } k=2 .\end{cases}
$$

One uses the fact that $H^{\mathrm{I}}(\Lambda, \Lambda)$ is the vector space of derivations of $\Lambda$ modulo the interior derivations.

\section{The theorem}

Throughout this note $k$ is an arbitrary field, $Q$ is a finite connected quiver and $F$ is the two sided ideal of $k Q$ generated by the arrows of $Q$.

Proposition. Let $\Lambda=k Q / F^{2}$. If $H^{2}(\Lambda, \Lambda)=0$ then $Q$ does not contain loops nor subquivers of the type

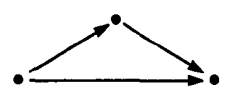

called 'unoriented triangles', and $Q$ is not the quiver $\rightleftarrows$ $\rightleftarrows$

Proof. Let $K=k((x))$ be the quotient power series field of $k[[x]]$, the power series ring in one variable.

If $Q$ contains a loop $b$, consider the two sided ideal $I$ of $K Q$ generated by $b^{2}-x b$ and by all the paths of length two except $b^{2}$. The algebra $\Lambda_{x}=K Q / I$ is a non-trivial one-parameter family of deformations of $\Lambda=k Q / F^{2}$, as defined in [7]. In fact $\Lambda_{x}$ is a $K$-algebra isomorphic to $K Q^{\prime} / F^{2}$, where $Q^{\prime}$ is obtained from $Q$ by deleting the loop $b$ and taking the disjoint union with a vertex. This algebra is clearly not isomorphic to $K Q / F^{2}$, and so $H^{2}(\Lambda, \Lambda) \neq 0$ using the result of [7] quoted in the introduction.

If $Q$ contains an unoriented triangle

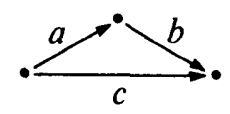

let $I$ be generated by $b a-x c$ and by all the paths of length two except $b a$. Again $\Lambda_{x}=K Q / I$ is a non-trivial one-parameter family of deformations of $\Lambda=k Q / F^{2}$. The $K$-algebra $\Lambda_{x}$ is isomorphic to $K Q^{\prime} / R$, where $Q^{\prime}$ is obtained from $Q$ by deleting the arrow $c$ and $R$ is generated by all the paths of length two except $b a$.

If

$$
Q=s \cdot \stackrel{a}{\stackrel{a}{\rightleftarrows}} \cdot t,
$$

let $I$ be the two sided ideal of $K Q$ generated by $b a-x s$ and $a b-x t$, and let $\Lambda_{x}$ be $K Q / I$. In fact $\Lambda_{x}$ is isomorphic to the algebra of $2 \times 2$ matrices over $K$. Notice that we cannot 
extend this deformation for $Q=\bullet \rightleftarrows \bullet \longrightarrow$ and $\Lambda=k Q / F^{2}$. In fact we shall see that $H^{2}(\Lambda, \Lambda)=0$ in this case.

The following lemma computes the dimension of $H^{2}(\Lambda, r)$ without the assumption that $H^{2}(\Lambda, \Lambda)=0$ (but still for a 2-nilpotent algebra).

LEMMA 1. Let $\Lambda=k Q / F^{2}$. Then

$$
\operatorname{dim}_{k} H^{2}(\Lambda, r)=\sum_{s, t \in Q_{0}} \sum_{u \in Q_{0}}\left|t Q_{1} s\right|\left|t Q_{1} u\right|\left|u Q_{1} s\right|
$$

Proof. Let $\gamma \in Q_{1}$, and denote by $s$ and $t$ its source and end vertices. The one dimensional vector space $k \gamma$ is a simple $\Lambda$-bimodule in the following way: $e \gamma=\delta_{e, t} \gamma$ and $\gamma e=\delta_{e, 8} \gamma$ for all $e$ in the $k$-basis $Q_{0} \cup Q_{1}$ of $\Lambda$.

We see that $r=\oplus_{\gamma \in Q_{1}} k \gamma$ is a direct decomposition of the semisimple $\Lambda$-bimodule $r$, and so

$$
H^{2}(\Lambda, r)=\underset{\gamma \in Q_{1}}{\oplus} H^{2}(\Lambda, k \gamma)
$$

Each of the simple $\Lambda$-bimodules $k \gamma$ is isomorphic to $\operatorname{Hom}_{k}(S, T)$, where $S$ and $T$ are the simple left $\Lambda$-modules corresponding to the vertices $s$ and $t$. We obtain

$$
H^{2}(\Lambda, r)=\bigoplus_{\gamma \in Q_{1}} H^{2}\left(\Lambda, \operatorname{Hom}_{k}(S, T)\right)
$$

We then use the following result [2, p. 179]. If $M$ and $N$ are left $\Lambda$-modules, the Hochschild cohomology of the $\Lambda$-bimodule $\operatorname{Hom}_{k}(M, N)$ can be obtained using the functor Ext over $\Lambda$ :

$$
H^{*}\left(\Lambda, \operatorname{Hom}_{k}(M, N)\right)=\operatorname{Ext}_{\Lambda}^{*}(M, N) .
$$

Then $H^{2}(\Lambda, r)=\oplus_{\gamma \in Q_{1}} \operatorname{Ext}_{\Lambda}^{2}(S, T)$.

The dimension of this vector space has been computed by $K$. Bongartz in [1, p. 463] using a particular projective resolution. In our case the ideal of relations is $F^{2}$ and so we obtain

$$
\begin{aligned}
\operatorname{dim}_{k} \operatorname{Ext}_{\lambda}^{2}(S, T) & =\operatorname{dim}_{k} t\left(F^{2} / F^{3}\right) s \\
& =\text { number of paths of length two from } s \text { to } t \\
& =\sum_{u \in Q_{0}}\left|t Q_{1} u\right|\left|u Q_{1} s\right|
\end{aligned}
$$

The number of $\operatorname{Ext} \lambda(S, T)$ direct summands in $H^{2}(\Lambda, r)$ is $\left|t Q_{1} s\right|$, and the formula is proved.

Using this lemma we can prove the converse of the proposition and obtain the following.

THEOREM. Let $\Lambda=k Q / F^{2}$, where $Q$ is connected. Then $H^{2}(\Lambda, \Lambda)=0$ if and only if $Q$ does not contain loops, or unoriented triangles and $Q$ is not the quiver $\rightleftarrows \bullet$.

Proof. If $Q$ is a quiver without loops and unoriented triangles, then Lemma 1 gives us that $H^{2}(\Lambda, r)=0$. The long exact sequence of cohomology associated to

gives

$$
0 \longrightarrow r \longrightarrow \Lambda \longrightarrow \Lambda / r \longrightarrow 0
$$

$$
0 \longrightarrow H^{2}(\Lambda, \Lambda) \longrightarrow H^{2}(\Lambda, \Lambda / r) \stackrel{\delta}{\longrightarrow} H^{3}(\Lambda, r) \longrightarrow \ldots
$$


Claim. For the quivers as in the theorem, the connecting homomorphism $\delta$ is injective; thus $H^{2}(\Lambda, \Lambda)=0$. If $Q$ is $\rightleftarrows \bullet$ then $1=\operatorname{dim}_{k} \operatorname{Ker} \delta=\operatorname{dim}_{k} H^{2}(\Lambda, \Lambda)$.

The rest of this note is devoted to the proof of the claim. We shall need a description of $H^{2}(\Lambda, \Lambda / r), H^{3}(\Lambda, r)$ and of the homomorphism $\delta$. The following lemma will give a description of $\delta$ using cup products. We choose to give it in a more general setting than just for 2-nilpotent algebras.

Let $\Lambda=k Q / I$, where $I$ is an admissible ideal. Let $r=F / I$ be the radical of $\Lambda$, and let $n$ be the nilpotence index of $\Lambda$, that is, $r^{n}=0$ and $r^{n-1} \neq 0$.

The product in $\Lambda$ gives bimodule homomorphisms

$$
r / r^{2} \otimes r^{n-2} / r^{n-1} \longrightarrow r^{n-1}, \quad r^{n-2} / r^{n-1} \underset{\Lambda}{\otimes} r / r^{2} \longrightarrow r^{n-1},
$$

which give cup products

$$
\begin{aligned}
& H^{1}\left(\Lambda, r / r^{2}\right) \otimes H^{i}\left(\Lambda, r^{n-2} / r^{n-1}\right) \longrightarrow H^{i+1}\left(\Lambda, r^{n-1}\right), \\
& H^{i}\left(\Lambda, r^{n-2} / r^{n-1}\right) \otimes H^{1}\left(\Lambda, r / r^{2}\right) \longrightarrow H^{i+1}\left(\Lambda, r^{n-1}\right),
\end{aligned}
$$

both denoted by $U$.

A set $P$ of oriented paths of $Q$ can be chosen such that $P$ is a basis of $\Lambda$, and such that the subset $P^{i}$ of oriented paths of length greater than or equal to $i$ is a basis of $r^{i}$. The set $Q_{1}$ of arrows is always in $P$, and is a basis of $r / r^{2}$.

To each arrow $a$ we can associate an element $f_{a} \in H^{1}\left(\Lambda, r / r^{2}\right)$ defined by $f_{a}(a)=a$ and $f_{a}(e)=0$ for all $e \in P, e \neq a$. It is easily checked that $f_{a}$ is a non-interior derivation. Let us call $\mathscr{S}$ the element $\Sigma_{a \in Q_{1}} f_{a} \in H^{1}\left(\Lambda, r / r^{2}\right)$.

Lemma 2. Let $\Lambda=k Q / I$, where $I$ is an admissible ideal of $k Q$. Consider the exact sequence

and

$$
0 \longrightarrow r^{n-1} \longrightarrow r^{n-2} \longrightarrow r^{n-2} / r^{n-1} \longrightarrow 0
$$

$$
\delta: H^{2 i}\left(\Lambda, r^{n-2} / r^{n-1}\right) \longrightarrow H^{3 i+1}\left(\Lambda, r^{n-1}\right)
$$

the connecting homomorphism.

Then $\delta(f)=\mathscr{S} \cup f+(-1)^{i+1} f \cup \mathscr{S}$.

Proof. Since $P^{n-1}$ is a $k$-basis of $r^{n-1}$ and $P^{n-2}$ of $r^{n-2}$, it follows that $P_{n-2}=P^{n-2} \backslash P^{n-1}$ is a $k$-basis of $r^{n-2} / r^{n-1}$. We choose the $k$-section $\sigma$ of the exact sequence corresponding to these bases, that is, $\sigma(\gamma)=\gamma$ if $\gamma \in P_{n-2}$.

In fact $r^{n-2} / r^{n-1}$ is a semisimple $\Lambda$-bimodule and $\oplus_{\gamma \in P_{n-2}} k \gamma$ is its decomposition. Thus, let $f: \Lambda^{\otimes i} \rightarrow k \gamma$ be an $i$-cocycle. This means that for all $x_{1}, \ldots, x_{i+1}$ in $P$ we have

$$
\begin{aligned}
\sum_{j-1}^{i}(-1)^{j} f\left(x_{1} \otimes \ldots \otimes\right. & \left.x_{j} x_{j+1} \otimes \ldots \otimes x_{i+1}\right) \\
& =-\left[x_{1} f\left(x_{2} \otimes \ldots \otimes x_{i}\right)+(-1)^{i+1} f\left(x_{1} \otimes \ldots \otimes x_{i}\right) x_{i+1}\right] .
\end{aligned}
$$

We know that $\delta(f)=d(\delta f)$, where $d$ is the Hochschild coboundary from $\operatorname{Hom}_{k}\left(\Lambda^{\otimes i}, r^{n-2}\right)$ to $\operatorname{Hom}_{k}\left(\Lambda^{\otimes i+1}, r^{n-2}\right)$. In fact $d(\sigma f) \in \operatorname{Hom}_{k}\left(\Lambda^{\otimes i+1}, r^{n-1}\right)$ and so we consider its cohomology class to obtain $\delta(f)$. Then we have

$$
\begin{aligned}
d(\gamma f)\left(x_{1} \otimes \ldots \otimes x_{i+1}\right)= & x_{1}(\sigma f)\left(x_{2} \otimes \ldots \otimes x_{i}\right) \\
& +\sum_{j=1}^{i}(-1)^{j}(\sigma f)\left(x_{1} \otimes \ldots \otimes x_{j} x_{j+1} \otimes \ldots \otimes x_{i+1}\right) \\
& +(-1)^{i+1}(\sigma f)\left(x_{1} \otimes \ldots \otimes x_{i}\right) x_{i+1} .
\end{aligned}
$$


Applying $\sigma$ to the relation (*) and substituting in the expression of $d(\sigma f)$, we obtain

$$
\begin{aligned}
d(\sigma f)\left(x_{1} \otimes \ldots \otimes x_{i+1}\right) & =x_{1} \sigma\left(f\left(x_{2} \otimes \ldots \otimes x_{i+1}\right)\right)-\sigma\left(x_{1} f\left(x_{2} \otimes \ldots \otimes x_{i+1}\right)\right) \\
& +(-1)^{i+1}\left[\sigma\left(f\left(x_{1} \otimes \ldots \otimes x_{i}\right)\right) x_{i+1}-\sigma\left(f\left(x_{1} \otimes \ldots \otimes x_{i}\right) x_{i+1}\right)\right]
\end{aligned}
$$

for all $x_{1}, \ldots, x_{i+1}$ in $P$.

Notice that the second and fourth terms of this sum are often zero: indeed, $f$ takes values in the simple $\Lambda$-bimodule $k \gamma$. But $\gamma$ is an oriented path of length $n-2$ from, say $s$ to $t$. Hence the only basis element with a non-zero action from the right is $s$, and from the left is $t$.

The first and third terms can only be non-zero if $x_{1}$ is an arrow or the vertex $t$ and $x_{i+1}$ is an arrow or the vertex $s$. Recall that $r^{n}=0$, thus if $x_{1}$ (or $x_{i+1}$ ) is of length greater than two then $x_{1} \gamma=0$ (or $\gamma x_{i+1}=0$ ), because the length of $\gamma$ is $n-2$.

If $x_{1}=t$, the value of the first term is equal to the value of the second term. If $x_{i+1}=s$, the second is equal to the fourth.

With these facts in mind, we can conclude the following:

$$
d(\sigma f)\left(x_{1} \otimes \ldots \otimes x_{i+1}\right)=0
$$

if both $x_{1}$ and $x_{i+1}$ are not arrows,

$$
=x_{1} \sigma\left(f\left(x_{2} \otimes \ldots \otimes x_{i+1}\right)\right)+(-1)^{i+1} \sigma\left(f\left(x_{1} \otimes \ldots \otimes x_{i}\right)\right) x_{i+1}
$$

if both $x_{1}$ and $x_{i+1}$ are arrows,

$$
=x_{1} \sigma\left(f\left(x_{2} \otimes \ldots \otimes x_{i+1}\right)\right)
$$

if $x_{1}$ is an arrow but not $x_{i+1}$,

$$
=(-1)^{i+1} \sigma\left(f\left(x_{1} \otimes \ldots \otimes x_{1}\right)\right) x_{i+1}
$$

if $x_{i+1}$ is an arrow but not $x_{1}$.

This description of $\delta f$ on the basis elements of $\Lambda^{\otimes i+1}$ coincides with the description of $\mathscr{S} \cup f+(-1)^{i+1} f \cup \mathscr{S}$, directly as cochains. In fact,

$$
\begin{aligned}
(\mathscr{S} \cup f)\left(x_{1} \otimes \ldots \otimes x_{i+1}\right) & =\sum_{a \in Q_{1}} f_{a}\left(x_{1}\right)(\sigma f)\left(x_{2} \otimes \ldots \otimes x_{i+1}\right) \\
& = \begin{cases}x_{1}(\sigma f)\left(x_{2} \otimes \ldots \otimes x_{i+1}\right) & \text { if } x_{1} \text { is an arrow, } \\
0 & \text { otherwise } .\end{cases}
\end{aligned}
$$

(Recall that $f_{a}(a)=a$ and $f_{a}(e)=0$ for all $e \in P, e \neq a$.)

We return now to the case of 2-nilpotent algebras, and give a description of $H^{2}(\Lambda, \Lambda / r)$ and $H^{3}(\Lambda, r)$. We assume that the quiver $Q$ does not contain loops or unoriented triangles. We could give a description without these assumptions, but it is simpler to work with them.

Let $E$ be the set of oriented paths of length two with same source and end vertex. More precisely $E=\amalg_{s, t \in Q_{0}}(s A t) \times(t A s)$, since $Q$ has no loops.

For each arrow $a$ from $s$ to $t$, let $L_{a}$ be the set of length-three oriented paths from $s$ to $t$, and $L=\amalg_{a \in A} L_{a}$.

We consider the vector spaces $k E$ and $k L$ with bases $E$ and $L$. Define a linear map $D: k E \rightarrow k L$ in the following way: for each $(b, a) \in(s A t) \times(t A s) \subset E$,

$$
D(b, a)=\sum_{x \in A s}(x b a)_{x}-\sum_{y \in s A}(b a y)_{y}
$$


where the index arrow means that $(x b a)_{x} \in L_{x}$; if there is a parallel arrow $x^{\prime}$ to $x$ (same source, same end) we have to make a distinction between elements of $L_{x}$ and $L_{x^{\prime}}$.

The $D$ we have just defined is the right model for $\delta$, as we see in the following.

LEMMA 3. There is an isomorphism between $H^{2}(\Lambda, \Lambda / r)$ and $k E$, and between $H^{3}(\Lambda, r)$ and $k L$ such that the diagram

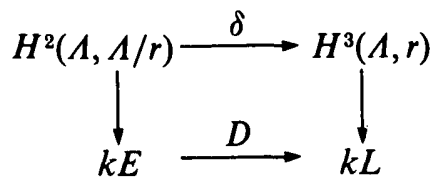

commutes.

Proof. Since $\Lambda / r=\oplus_{s \in Q_{0}} k s$ as bimodules, $H^{2}(\Lambda, \Lambda / r)=\oplus_{s \in Q_{0}} H^{2}(\Lambda, k s)$.

Let $(b, a) \in(s A t) \times(t A s)$, and consider $f_{(b, a)}: \Lambda \otimes \Lambda \rightarrow k s$ defined by

$$
f_{(b, a)}(b \otimes a)=s
$$

and $f_{(b, a)}$ gives zero when evaluated in any other tensor product of two basis vectors of $\Lambda$. These linear maps are easily checked to be 2-cocycles and they are linearly independent modulo coboundaries: if $g: \Lambda \rightarrow k s$ is a linear map, then

$$
(d g)(b \otimes a)=b g(a)-g(b a)+g(b) a=0
$$

because each term in the sum is zero ( $k s$ is a semisimple bimodule and $b a \in r^{2}=0$ ); thus if $\sum \lambda_{b a} f_{(b, a)}=d g$, we obtain $\lambda_{b a}=0$ for each $(b, a) \in s A t \times t A s$.

Moreover, the set $\left\{f_{(b, a)}\right\}_{(b, a) \in E}$ is a basis of $H^{2}(\Lambda, k s)$ because its cardinality is the dimension of $H^{2}(\Lambda, k s)$. Indeed,

$$
H^{2}(\Lambda, k s)=H^{2}\left(\Lambda, \operatorname{Hom}_{k}(S, S)\right)=\operatorname{Ext}(S, S) ;
$$

the dimension of $\operatorname{Ext}_{\lambda}(S, S)$ is the number of paths of length two from $s$ to $s$ by Bongartz's result [1, p. 463].

Thus the left vertical isomorphism is obtained by sending $f_{(b, a)}$ to $(b, a)$.

To obtain the one on the right consider $r=\oplus_{a \in A} k a$ as a bimodule. Hence $H^{3}(\Lambda, r)=\oplus_{a \in A} H^{3}(\Lambda, k a)$.

Let $\left(c_{3} c_{2} c_{1}\right)_{a} \in L_{a}$ be a path of length three from the source $s$ of $a$ to its end $t$. Define $f_{c_{3} c_{2} c_{1}}^{a}: \Lambda^{\otimes 3} \rightarrow k a$ by $f_{c_{3} c_{2} c_{1}}^{a}\left(c_{3} \otimes c_{2} \otimes c_{1}\right)=a$ and to be zero evaluated in any other tensor product of three basis elements of $\Lambda$. These linear maps are 3-cocycles, and linearly independent modulo coboundaries: if $g: \Lambda \otimes \Lambda \rightarrow k a$ is a linear map,

$$
\begin{aligned}
d g\left(c_{3} \otimes c_{2} \otimes c_{1}\right) & =c_{3} g\left(c_{2} \otimes c_{1}\right)-g\left(c_{3} c_{2} \otimes c_{1}\right)+g\left(c_{3} \otimes c_{2} c_{1}\right)-g\left(c_{3} \otimes c_{2}\right) c_{1} \\
& =0,
\end{aligned}
$$

again because $k a$ is a semisimple bimodule and $r^{2}=0$. Thus if a $k$-linear combination of the $f_{c_{3} c_{2} c_{1}}^{a}$ is equal to a coboundary, then all the coefficients are zero.

The $f_{c_{3} c_{2} c_{1}}^{a}$ are in the right number to be a basis of $H^{3}(\Lambda, k a)$, because

$$
\operatorname{dim}_{k} H^{3}(\Lambda, k a)=\operatorname{dim}_{k} \operatorname{Ext}_{\Lambda}^{3}(S, T)=\operatorname{dim}_{k} t\left(F^{3} / F^{4}\right) s
$$

(the last equality is derived from [1, p. 462, last line] with $n=1, J=F, I=F^{2}$ ). But $\operatorname{dim}_{k} t\left(F^{3} / F^{4}\right) s$ is the number of length-three oriented paths from $s$ to $t$, which is $\left|L_{a}\right|$.

The isomorphism between $H^{3}(\Lambda, r)$ and $k L$ is obtained sending $f_{c_{3} c_{2} c_{1}}^{a}$ to $\left(c_{3} c_{2} c_{1}\right)_{a}$. 
By composing these isomorphisms and $\delta$, and using the description of $\delta$ in terms of cup products, we get exactly the defined $D$. We need only check that

$$
f_{x} \cup f_{(b, a)}=f_{x b a}^{x},
$$

which is already true at the level of cochains.

We can now return to the claim which proves the theorem. Namely $D: k E \rightarrow k L$ is injective when $Q$ is connected, does not contain loops or unoriented triangles, and $Q$ is not the quiver $\rightleftharpoons \bullet$.

Suppose that $\dot{\Sigma}_{(b, a) \in E} \lambda_{(b, a)} D(b, a)=0$. Recall that

$$
D(b, a)=\sum_{x \in A s}(x b a)_{x}-\sum_{y \in s A}(b a y)_{y}
$$

if $(b, a) \in s A t \times t A s$.

The central remark is the following: for each $(b, a)$ there exist $x \in A s, x \neq a$ (or $y \in s A, y \neq b)$. Indeed, $Q$ is not the quiver $\rightleftharpoons \cdot$ and $Q$ is connected. Thus $(x b a)_{x}$ (or $\left.(\text { bay })_{y}\right)$ is a component of $D(b, a)$, but not a component of any other $D(d, c)$ : if it were, then $(x b a)_{x}=(z d c)_{z}$ or $(x b a)_{x}=(d c z)_{z}$ for some arrow $z$. The first equality is impossible since we suppose that $(b, a) \neq(d, c)$. The second would imply that $x=z=a$, and so $x=a$, which is a contradiction. The argument is the same if $(\text { bay })_{y}$ is a component of $D(b, a)$.

Using this remark we see that replacing each $D(b, a)$ by its value in the linear dependence relation, we get a linear combination of the basis $L$, such that for each $(b, a) \in E$ there exists $x$ (or $y$ ) with the coefficient of $(x b a)_{x}$ (or $\left.(b a y)_{y}\right)$ equal to $\lambda_{(b, a)}$. Hence $\lambda_{(b, a)}=0$ and $D$ is injective.

In contrast with this, the matrix of $D$ for $Q=\bullet \rightleftarrows \bullet$ is

$$
\left[\begin{array}{rr}
1 & -1 \\
-1 & 1
\end{array}\right]
$$

and so $\operatorname{dim}_{k} H^{2}\left(k Q / F^{2}, k Q / F^{2}\right)=1$ in this case.

We conclude this note with a remark.

In [3] we gave sufficient conditions on a basic and connected finite dimensional algebra $\Lambda$ for the vanishing of $H^{2}(\Lambda, \Lambda)$. Let $Q$ be a connected quiver, let $I$ be an admissible ideal of $k Q$, and let $\Lambda=k Q / I$. If for all couples of vertices $(s, t)$ such that $t \Lambda s \neq 0$ we have $t I s=t(F I+I F) s$ then $H^{2}(\Lambda, \Lambda)=0$.

These conditions are not necessary: when

$$
Q=\bullet \rightleftarrows \bullet \longrightarrow \cdot I=F^{2} \text {, and } \Lambda=k Q / F^{2},
$$

we have $s \Lambda s \neq 0$, and $s F^{2} s \neq s F^{3} s$. At the same time we know from this note that $H^{2}(\Lambda, \Lambda)=0$.

But if $Q$ has no oriented cycles and $I=F^{2}$, the conditions of [3] are equivalent to the fact that $Q$ does not contain unoriented triangles. From the above theorem we know that this is necessary and sufficient for $H^{2}\left(k Q / F^{2}, k Q / F^{2}\right)$ to be zero, when $Q$ has no oriented cycles. 


\section{References}

1. K. BongartZ, 'Algebras and quadratic forms', J. London Math. Soc. (2) 28 (1983) 461-469.

2. H. CARTAN and S. Eilenderg, Homological algebra (University Press, Princeton, 1956).

3. C. CibiLs, 'Cohomologie de Hochschild d'algebres de dimension finie', Comm. Algebra to appear.

4. P. GABRIEL, 'Indecomposable representations II', Symposia Matematica 2 (Istituto Nazionale di alta Matematica, Roma, 1973) 81-104.

5. $P$. GABRIEL, 'Auslander-Reiten sequences and representation finite algebras', Representation theory $I$, proceedings, 1979, Lecture Notes in Mathematics 831 (Springer, Berlin, 1980) 1-71.

6. P. GABRIEL, 'Finite representation type is open', Representations of algebras, Ottawa 1974, Lecture Notes in Mathematics 488 (Springer, Berlin, 1975) 132-155.

7. M. Gerstenhaber, 'On the deformations of rings and algebras', Ann. of Math. 79 (1964) 59-103.

Section de Mathématiques

Université de Genève

C.P. 240

CH-1211 Genève 24

Switzerland
Ecole Normale Supérieure

Equipe des groupes finis

1 rue Maurice Arnoux

F-92140 Montrouge

France 